\title{
Detecting Ultra-Violet Radiation by Using Titanium Dioxide Nanoparticles
}

\author{
Naser Hatefi Kargan, Mousa Aliahmad, Samereh Azizi \\ Department of Physics, Faculty of Sciences, University of Sistan and Baluchestan, Zahedan, Iran. \\ Email: aliahmad@phys.usb.ac.ir
}

Received January $24^{\text {th }}, 2012$; revised March $2^{\text {nd }}, 2012$; accepted March $14^{\text {th }}, 2012$

\begin{abstract}
In this paper sensitivity of chemically synthesized titanium dioxide $\left(\mathrm{TiO}_{2}\right)$ nanoparticles to ultra-violet (UV) radiation is investigated. For this purpose three types of ultra-violet photodetectors were fabricated, one with as-synthesized $\mathrm{TiO}_{2}$ nanoparticles, another one with $\mathrm{TiO}_{2}$ nanoparticles annealed at $600^{\circ} \mathrm{C}$, and the third one with $\mathrm{TiO}_{2}$ nanoparticles annealed at $800^{\circ} \mathrm{C}$. In each case the annealing time duration was one hour. The photodetectors were fabricated on glass slides where on them a thin layer of fluorine doped tin oxide (FTO) was deposited by spray pyrolysis. The results show that all photodetectors are sensitive to UV radiation where the photodetectors fabricated from annealed $\mathrm{TiO}_{2}$ nanoparticles are more sensitive than the photodetectors fabricated from as-synthesized $\mathrm{TiO}_{2}$ nanoparticles.
\end{abstract}

Keywords: Ultra-Violet Photodetector; Titanium Dioxide; Nanocrystals; Nanoparticles

\section{Introduction}

In recent years, UV photodetectors have got much consideration due to their civilian and military applications. These photodetectors are used in UV radiation measurement equipment, space research, detecting missiles at early stages of their launch, astronomy and biology $[1,2]$. In general, UV photodetectors are fabricated from large band gap inorganic semiconductor materials such as: $\mathrm{GaN}[3,4], \mathrm{CdS}$ [5], diamond [6], or Si [7,8]. These materials are produced using complicated processing steps that are costly and are not appropriate for large scale applications. Recently semiconductor nanoparticles synthesized by chemical methods have been studied where in comparison to the semiconductor materials produced by previous techniques have advantages such as: low cost, simple preparation methods, capable of large scale applications, and vast options on substrate choice.

$\mathrm{TiO}_{2}$ is a wide band gap semiconductor material which can be used for UV photodetector fabrication [9]. This material crystalizes in three major phases: rutile, anatase, and brookite [10]. Rutile phase is thermodynamically more stable than the two other phases. Rutile $\mathrm{TiO}_{2}$ has direct and indirect band gaps. Its direct band gap makes $\mathrm{TiO}_{2}$ useful for photodetector fabrication. In this research $\mathrm{TiO}_{2}$ rutile phase nanoparticles were synthesized by a chemical method. Then by using these nanoparticles ultra-violet photodetectors were fabricated that are sensitive to ultra-violet radiation.

\section{Synthesis of $\mathrm{TiO}_{2}$ Nanoparticles}

We synthesized $\mathrm{TiO}_{2}$ nanoparticles by using $20 \mathrm{~mL}$ titanium (III) chloride solution (15\%) in 10\% hydrochloric acid from Merck Company. The solution was stirred vigorously in a reaction flask for $20 \mathrm{~min}$ at room temperature. Then $0.7 \mathrm{~mL}$ ammonia solution $(25 \%)$ from Merck was diluted by $57.3 \mathrm{~mL}$ double distilled water and this $60 \mathrm{~mL}$ solution was added to the reaction flask drop wise very slowly (during 5 hours). Then the solution was left for 48 hours while it was stirred vigorously; during this time color of the solution gradually turns to white. White precipitation settle done properly. We centrifuged and washed the precipitation with water and ethanol a few times. This centrifuging and washing was repeated for three times.

We prepared three types of $\mathrm{TiO}_{2}$ samples, as-synthesized (sample A), annealed at $600^{\circ} \mathrm{C}$ (sample B), and annealed at $800^{\circ} \mathrm{C}$. Duration of annealing time was one hour for both sample B and sample C.

\section{Fabrication of Photodetectors}

For fabricating UV photodetectors fluorine doped tin oxide (FTO) was coated on glass substrates by spray pyrolysis. For depositing FTO, 33.3 gr tin tetrachloride pentahydrate $\left(\mathrm{SnCl}_{4.5} \mathrm{H}_{2} \mathrm{O}, 98 \%\right)$ was dissolved in $50 \mathrm{~mL}$ double distilled water by using magnetic stirrer for a few minutes. Then $50 \mathrm{~mL}$ ethanol was added to the solution. Addition of alcohol turns the solution slightly blurred 
where by adding a few droplets of hydrochloric acid $\mathrm{pH}$ of the solution decreases. It leads to increasing of the solution solubility which causes the solution become clear again. Then to this solution we added $0.5 \mathrm{gr} \mathrm{NH}_{4} \mathrm{~F}$ $(99.99 \%) .10 \mathrm{~mL}$ of this solution was sprayed on glass slides where they were heated to $450^{\circ} \mathrm{C}$. The solution was sprayed at $2 \mathrm{~atm}$ air pressure with the speed of 10 $\mathrm{mL} / \mathrm{min}$ where the spraying nozzle opening diameter was $0.3 \mathrm{~mm}$ and its distance from substrates was $35 \mathrm{~cm}$. The deposited FTO layer had $200 \mathrm{~nm}$ thickness and its sheet resistance was $484 \Omega$.

After coating, FTO conducting oxide was disconnected by scribing a scribe line all along the width of glass slides. The scribe line was created by a diamond tip glass scriber and the width of the scribed line was $90 \mu \mathrm{m}$. Then $\mathrm{TiO}_{2}$ nanoparticles dispersed in diethylene glycol (50\% by weight), and spin coated on the FTO film at $1000 \mathrm{rpm} / \mathrm{min}$. $\mathrm{TiO}_{2}$ coating was done on the scribed line where the coating area was $5 \mathrm{~mm}$ by $5 \mathrm{~mm}$. Then they are placed in an oven for $30 \mathrm{~min}$ and the oven temperature was $450^{\circ} \mathrm{C}$.

Three types of photodetectors were fabricated, one type with as synthesized $\mathrm{TiO}_{2}$ nanoparticles (type A), another one with $\mathrm{TiO}_{2}$ nanoparticles annealed at $600^{\circ} \mathrm{C}$ (type $\mathrm{B}$ ), and the other with $\mathrm{TiO}_{2}$ nanoparticles annealed at $800^{\circ} \mathrm{C}$ (type $\mathrm{C}$ ).

\section{Characterization of $\mathrm{TiO}_{2}$ Nanoparticles}

Some techniques were used for analyzing $\mathrm{TiO}_{2}$ nanoparticles such as: X-ray diffraction spectroscopy (XRD), scanning electron microscopy (SEM), and UV-VIS spectroscopy. Figure 1 shows UV-VIS spectrum of the samples. As Figure 1 shows by increasing annealing temperature absorption at peak point is shifted to longer

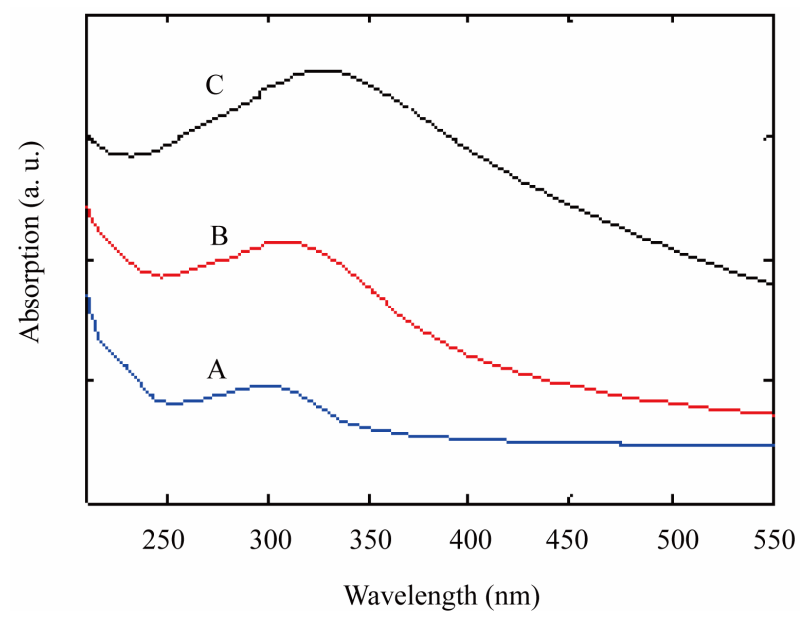

Figure 1. UV-VIS spectrum of $\mathrm{TiO}_{2}$ nanoparticles samples: as-synthesized $\mathrm{TiO}_{2}$ nanoparticles (A); $\mathrm{TiO}_{2}$ nanoparticles annealed at $600^{\circ} \mathrm{C}(\mathrm{B})$ and $\mathrm{TiO}_{2}$ nanoparticles annealed at $800^{\circ} \mathrm{C}(\mathrm{C})$. wavelengths (red shift) which indicates increasing nanoparticles size by increasing annealing temperature.

Figure 2 shows XRD spectrum of the samples. The Xray wavelength used for measurements was $0.154 \mathrm{~nm}$. It

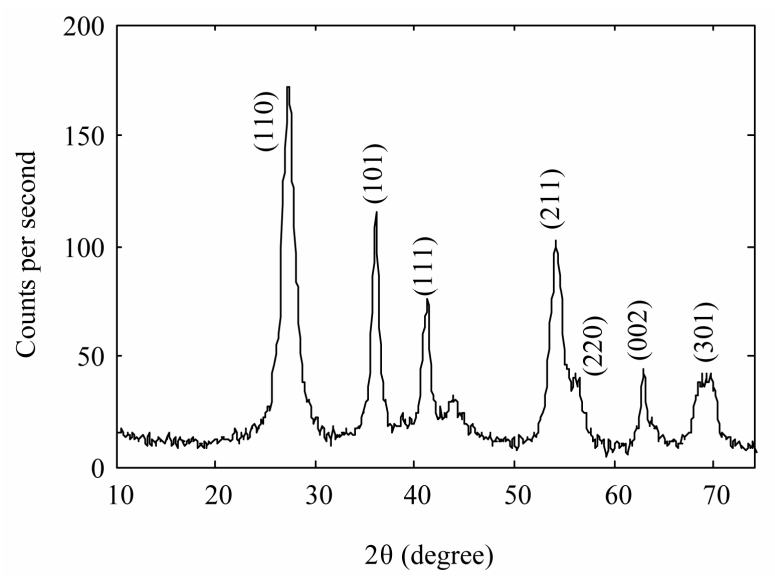

(a)

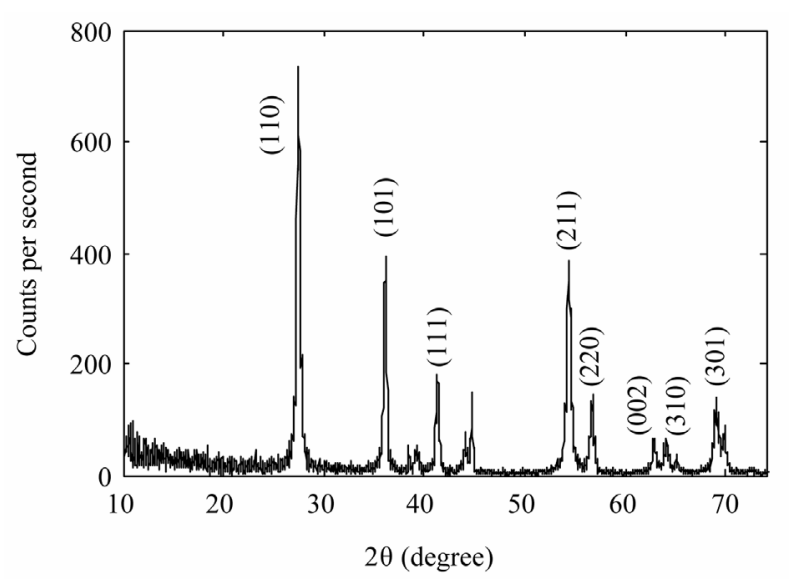

(b)

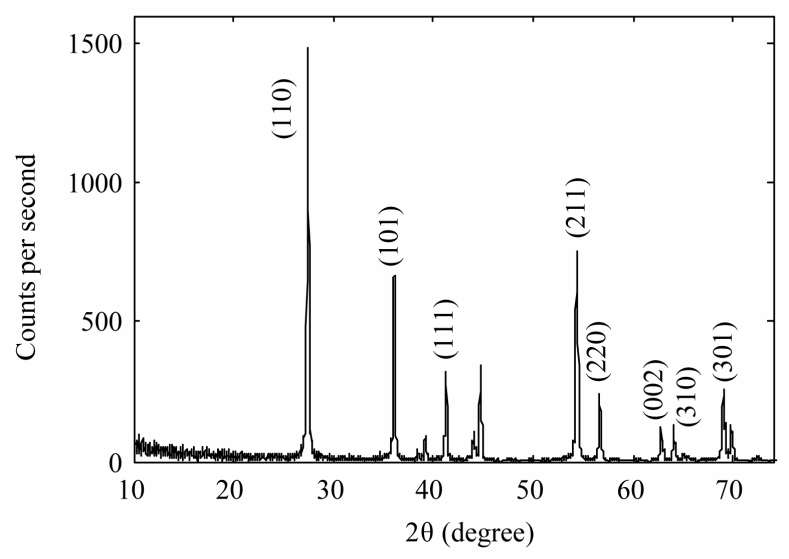

(c)

Figure 2. XRD spectrum of $\mathrm{TiO}_{2}$ nanoparticles samples: as-synthesized $\mathrm{TiO}_{2}$ nanoparticles (a); $\mathrm{TiO}_{2}$ nanoparticles annealed at $600^{\circ} \mathrm{C}$ (b) and $\mathrm{TiO}_{2}$ nanoparticles annealed at $800^{\circ} \mathrm{C}$ (c). 
is evident from the figure that as synthesized nanoparticles are in rutile phase. By increasing annealing temperature the intensity of the peaks increase which could be due to an increase in the size of as-synthesized nanoparticles, or may be the nanoparticles get more crystalized. We used Scherrer's formula for average grain size of nanoparticles. By calculating it has been found $10 \mathrm{~nm}$, $39 \mathrm{~nm}$, and $104 \mathrm{~nm}$ for the samples $\mathrm{A}, \mathrm{B}$, and $\mathrm{C}$ respectively.

SEM images of the nanoparticles (Figure 3) show that they are approximately in spherical form and their sizes are approximately the same $(57 \mathrm{~nm})$ for samples A, B, and $\mathrm{C}$. The SEM result is in contradiction with the grain size of particles which we have found from XRD patterns, and UV-VIS spectrum of the samples that indicate increase in nanoparticles size with increasing annealing temperature. Thus this contradiction indicates that the size of nanoparticles does not change by annealing, but they get more crystalized. In other words, as-synthesized $\mathrm{TiO}_{2}$ nanoparticles are more in polycrystalline phase and they change more and more to monocrystalline phase by increasing annealing temperature.

\section{Characterization of Photodetectors}

We did some measurements on the fabricated detectors. First of all we measured dark current, and photocurrent (at constant UV illumination power) of the fabricated photodetectors. Then we measured photocurrent of the samples as a function of illumination power at a constant applied voltage. Dark current measurements were done at room temperature where the samples were kept inside a closed box. Figure 4 shows dark current of the samples as a function of applied voltage. It is evident from the figure that the photodetectors fabricated from annealed $\mathrm{TiO}_{2}$ nanoparticles have higher dark currents than the photodetector fabricated from as-synthesized $\mathrm{TiO}_{2}$. This is due to the fact that annealed $\mathrm{TiO}_{2}$ nanoparticles are more crystalline, so their conductivity is increased as well.

Photocurrent measurements were done by placing the photodetectors inside a box where the devices were illuminated by an Hg 100 UV lamp (from Leybold) through a $2 \mathrm{~cm}$ diameter hole on the box. For measuring photocurrent as a function of voltage at constant illumination power, the UV lamp was kept at a constant distance (14 $\mathrm{cm})$ from the devices. Photocurrent measurement as a function of illumination power at constant applied bias $(10 \mathrm{~V})$ was done by varying the UV lamp distance from photodetectors. Figure 5 shows the results. It is clear from the figure that sensitivity of the devices fabricated from annealed $\mathrm{TiO}_{2}$ nanoparticles increase with the annealing temperature. Although with increasing annealing temperature dark current increases as well, this is not a

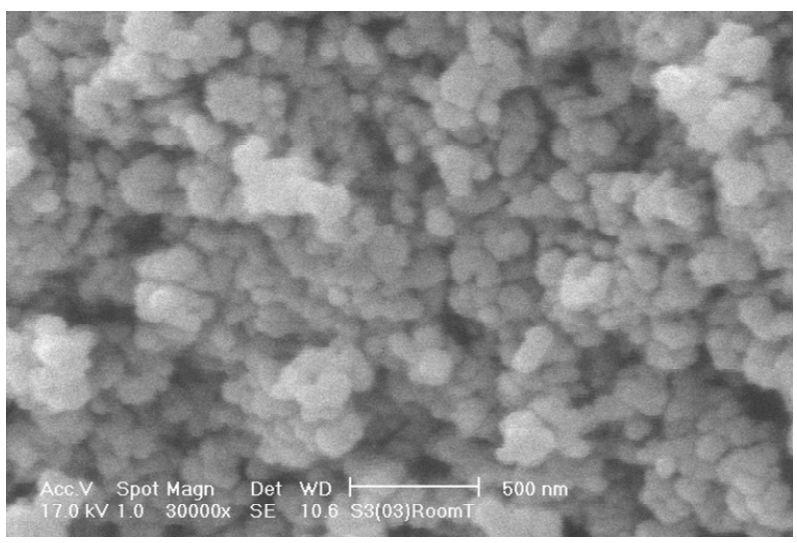

(a)

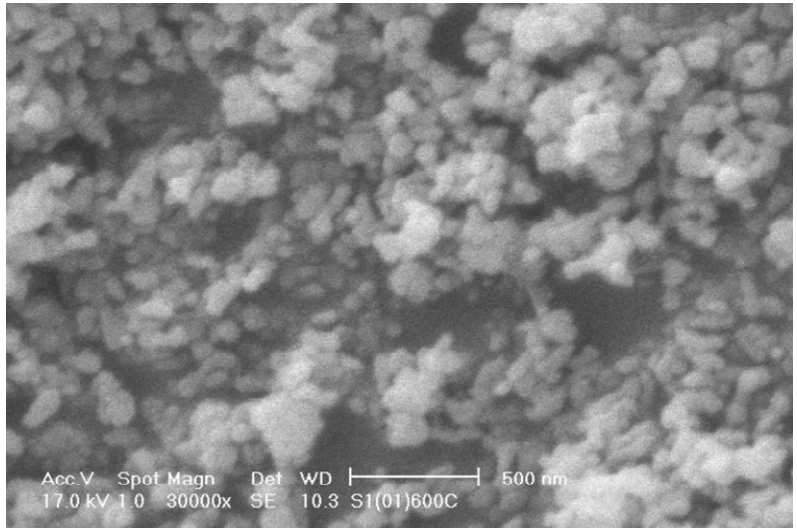

(b)

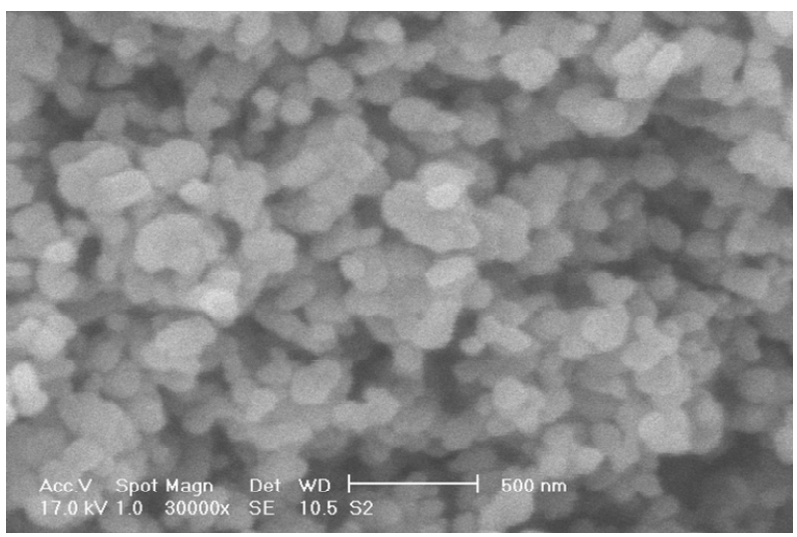

(c)

Figure 3. SEM image of $\mathrm{TiO}_{2}$ nanoparticles samples: assynthesized $\mathrm{TiO}_{2}$ nanoparticles (a); $\mathrm{TiO}_{2}$ nanoparticles annealed at $600^{\circ} \mathrm{C}$ (b) and $\mathrm{TiO}_{2}$ nanoparticles annealed at $800^{\circ} \mathrm{C}$ (c).

disadvantage. This is due to the fact that photocurrent increases a few times more than dark current. For example if dark current and photocurrent for samples A and C are compared, it can be obtained that while dark current increases by about two times, photocurrent increases by about 8 times. 


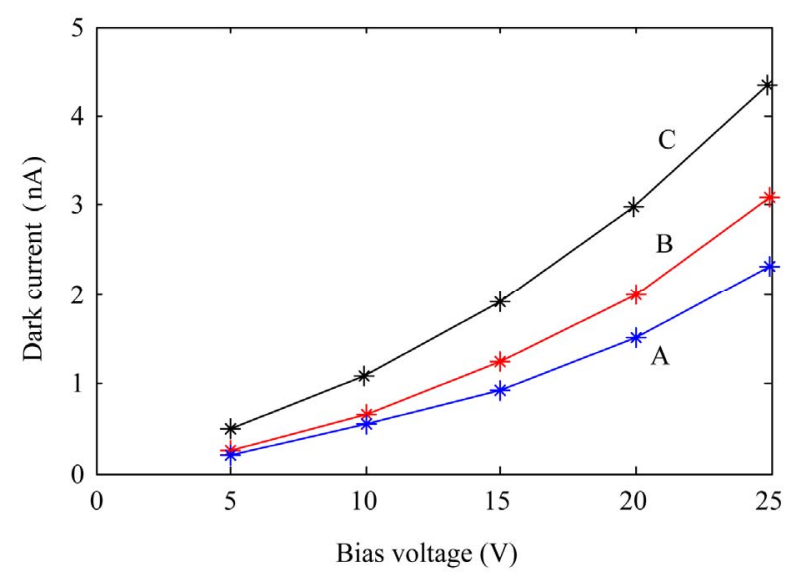

Figure 4. Dark current of the fabricated photodetectors: fabricated from as-synthesized $\mathrm{TiO}_{2}$ nanoparticles (A); $\mathrm{TiO}_{2}$ nanoparticles annealed at $600^{\circ} \mathrm{C}(\mathrm{B})$ and $\mathrm{TiO}_{2}$ nanoparticles annealed at $800^{\circ} \mathrm{C}(\mathrm{C})$.

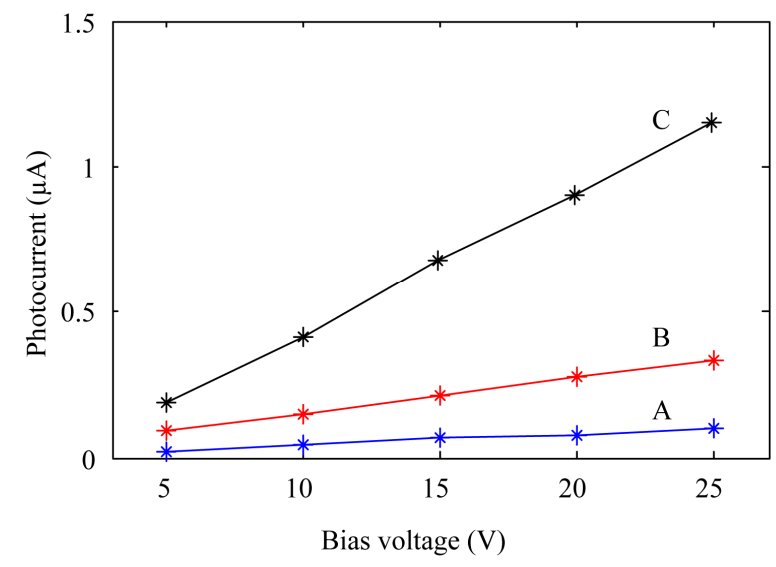

(a)

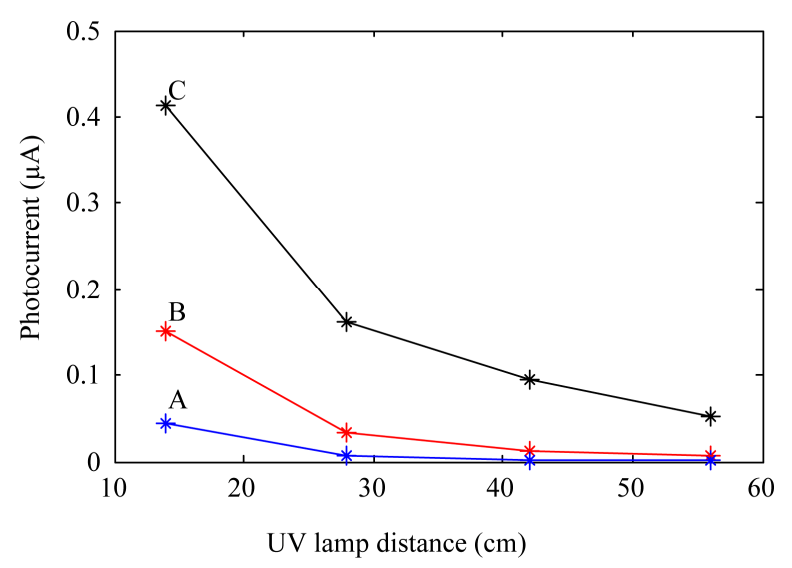

(b)

Figure 5. Photocurrent of the fabricated photodetectors: fabricated from as-synthesized $\mathrm{TiO}_{2}$ nanoparticles (A); $\mathrm{TiO}_{2}$ nanoparticles annealed at $600^{\circ} \mathrm{C}(\mathrm{B})$; and $\mathrm{TiO}_{2}$ nanoparticles annealed at $800^{\circ} \mathrm{C}$ (C). (a) shows photocurrent at a constant illumination power, and (b) shows photocurrent as a function of UV lamp distance from the devices at a constant applied bias.

\section{Conclusions}

We synthesized $\mathrm{TiO}_{2}$ nanoparticles by a chemical method and annealed them at $600^{\circ} \mathrm{C}$, and $800^{\circ} \mathrm{C}$ where the annealing time duration was one hour. SEM, UV-VIS, and XRD results show that as synthesized nanoparticles are in rutile phase and the nanoparticles get more crystalline by annealing at higher temperatures.

We fabricated photodetectors by using as-synthesized and annealed $\mathrm{TiO}_{2}$ nanoparticles. Measurements show that the sensitivity of the devices increase with increasing annealing temperature of $\mathrm{TiO}_{2}$ nanoparticles which from them the detectors were fabricated. This confirms the result obtained from UV-VIS, SEM and XRD measurements that annealing causes $\mathrm{TiO}_{2}$ nanoparticles to get more crystalline.

\section{REFERENCES}

[1] E. Monroy, F. Omnes and F. Calle, "Wide-Bandgap Semiconductor Ultraviolet Photodetectors," Semiconductor Science and Technology, Vol. 18, No. 4, 2003, pp. R33R51. doi:10.1088/0268-1242/18/4/201

[2] S. V. Averine, P. I. Kuznetzov, V. A. Zhitov and N. V. Alkeev, "Solar-Blind MSM-Photodetectors Based on $\mathrm{Al}_{\mathrm{x}} \mathrm{Ga}_{1-\mathrm{x}} \mathrm{N} / \mathrm{GaN}$ Heterostructures Grown by MOCVD," Solid-State Electronics, Vol. 52, No. 5, 2008, pp. 618624. doi:10.1016/i.sse.2007.10.037

[3] S. K. Zhang, W. B. Wang, I. Shtau, F. Yun, L. He, H. Morkoc, X. Zhou, M. Tamargo and R. R. Alfano, "Backilluminated GaN/AlGaN Heterojunction Ultraviolet Photodetector with High Internal Gain," Applied Physics Letters, Vol. 81, No. 25, 2002, pp. 4862-4864. doi:10.1063/1.1526166

[4] I.-S. Seo, I.-H. Lee, Y.-J. Park and C.-R. Lee, "Characteristics of UV Photodetector Fabricated by $\mathrm{Al}_{0.3} \mathrm{Ga}_{0.7} \mathrm{~N} /$ GaN Heterostructure," Journal of Crystal Growth, Vol. 252, No. 1-3, 2003, pp. 51-57. http://dx.doi.org/10.1016/S0022-0248(02)02523-X

[5] M. S. Shur, S. Rumyantsev, R. Gaska, B. Q. Wei, R. Vajtai, P. M. Ajayan and J. Sinius, "Structural and Transport Properties of CdS Films Deposited on Flexible Substrates," Solid-State Electronics, Vol. 46, No. 9, 2002, pp. 1417-1420. doi:10.1016/S0038-1101(02)00073-4

[6] S. Salvatori, G. Mazzeo, G. Conte, M. C. Rossi and V. Ralchenko, "Polycrystalline Diamond Position Sensitive Detector for Excimer Laser UV Radiation," Diamond and Related Materials, Vol. 13, No. 4-8, 2004, pp. 948-953.

[7] R. F. Wolffenbuttel, "Silicon Photodetectors with a Selective Spectral Response," Sensors Update, Vol. 9, No. 1, 2001, pp. $69-101$. doi:10.1002/1616-8984(200105)9:1<69::AID-SEUP69>3 .0.CO;2-Q

[8] D. Caputo, G. de Cesare, A. Nascetti and M. Tucci, "On the Fabrication and Characterization of Amorphous Silicon Ultra-Violet Sensor Array," Thin Solid Films, Vol. 517, No. 23, 2009, pp. 6422-6425. doi:10.1016/j.tsf.2009.02.064 
[9] X. Kong, C. Liu, W. Dong, X. Zhang, C. Tao, L. Shen, J. Zhou, Y. Fei and S. Ruan, "Metal-Semiconductor-Metal $\mathrm{TiO}_{2}$ Ultraviolet Detectors with Ni Electrodes," Applied Physics Letters, Vol. 94, No. 12, 2009, Article ID: 123502. doi: $10.1063 / 1.3103288$
[10] X. Chen and S. S. Mao, "Titanium Dioxide NanoMaterials: Synthesis, Properties, Modifications, and Applications," Chemical Reviews, Vol. 107, No. 7, 2007, pp. 2891-2959. doi:10.1021/cr0500535 